\title{
Guillaume Cuchet, Les Voix d'outre-tombe. Tables tournantes, spiritisme et société au XIXe siècle
}

Paris, Éditions du Seuil, coll. «L’Univers historique », 2012, 457 p.

\section{Agnès Desmazières}

\section{CpenEdition}

Journals

Édition électronique

URL : http://journals.openedition.org/assr/25447

DOI : $10.4000 /$ assr.25447

ISSN : $1777-5825$

Éditeur

Éditions de l'EHESS

Édition imprimée

Date de publication : 30 décembre 2013

Pagination : 169

ISSN : 0335-5985

Référence électronique

Agnès Desmazières, « Guillaume Cuchet, Les Voix d'outre-tombe. Tables tournantes, spiritisme et société au xıxe siècle ", Archives de sciences sociales des religions [En ligne], 164 | 2013, mis en ligne le 19 février 2014, consulté le 21 septembre 2020. URL : http://journals.openedition.org/assr/25447 ; DOI : https://doi.org/10.4000/assr.25447

Ce document a été généré automatiquement le 21 septembre 2020.

(C) Archives de sciences sociales des religions 


\section{Guillaume Cuchet, Les Voix d'outre- tombe. Tables tournantes, spiritisme et société au XIXe siècle}

Paris, Éditions du Seuil, coll. «L'Univers historique », 2012, 457 p.

Agnès Desmazières

\section{RÉFÉRENCE}

Guillaume Cuchet, Les Voix d'outre-tombe. Tables tournantes, spiritisme et société au XIXe siècle, Paris, Éditions du Seuil, coll. «L'Univers historique », 2012, 457 p. 
1 L'histoire du spiritisme constitue un terrain privilégié tant pour l'analyse des reconfigurations des rapports entre science et religion au XIX ${ }^{\mathrm{e}}$ siècle, que pour l'étude des échanges culturels entre Europe et Amérique du Nord et, plus largement, des mutations sociales qui les traversent. D’origine américaine et participant $\mathrm{du}$ « renouveau religieux » qui s'affirme «aux marges du protestantisme » (Ann Taves, Fits, Trances, and Visions: Experiencing Religion and Explaining Experience from Wesley to James, Princeton, Princeton University Press, 1999, p. 167), le spiritisme se diffuse à partir du début des années 1850 sur le continent européen où il se confronte au catholicisme dominant.

Il n'est donc pas surprenant qu'en dépit $\mathrm{du}$ travail pionnier et fondamental de Nicole Edelman (Voyantes, guérisseuses et

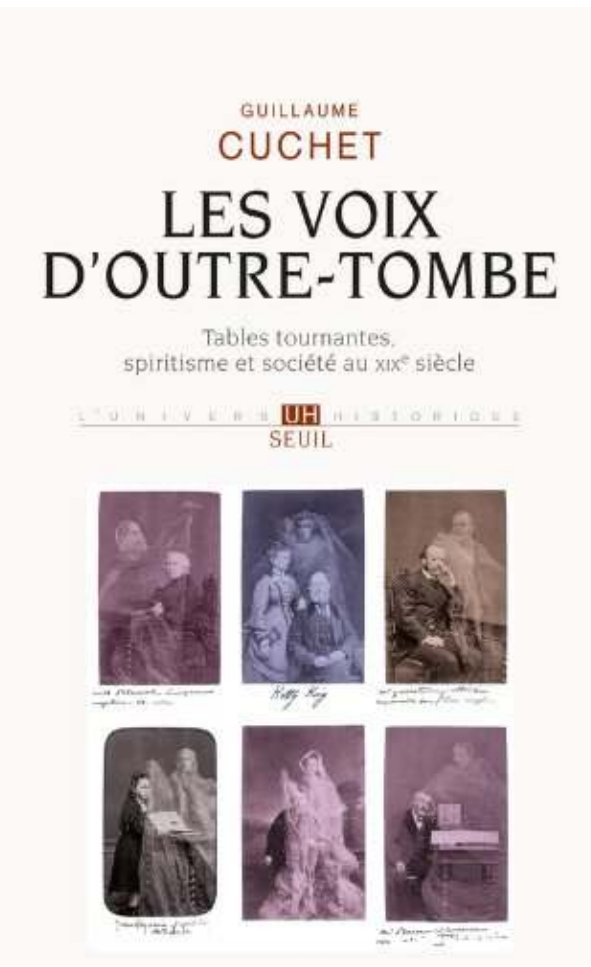
visionnaires en France, 1785-1914, Paris, Albin Michel, 1995), l'historiographie américaine prévale en ce domaine. Après avoir largement défriché le champ du spiritisme nordaméricain, les historiens anglo-saxons ont porté, de manière croissante, depuis une décennie, leur attention sur le cas français selon deux directions principales. La première perspective, qui s'inscrit dans le cadre d'une histoire culturelle et sociale du religieux, s'intéresse, à la suite des travaux de Thomas Kselman, au retour de la "religion populaire» dans la France du XIX ${ }^{\mathrm{e}}$ siècle, duquel le spiritisme participe (notamment Lynn L. Sharp, Secular Spirituality: Reincarnation and Spiritism in NineteenthCentury France, Lanham - Plymouth, Lexington Books, 2006).

3 La seconde, plus ample, participe du développement de l'histoire de la connaissance et de l'épistémologie scientifique. Comment situer l'apparition du spiritisme face à l'affirmation, au $\mathrm{XIX}^{\mathrm{e}}$ siècle, d'une culture de la rationalité et de l'objectivité scientifique? En quoi reflète-t-elle l'émergence de nouvelles formes d'« investigation du surnaturel»? (Sofie Lachapelle, Investigating the Supernatural: From Spiritism and Occultism to Psychical Research and Metapsychics in France, 1853-1931, Baltimore, The John Hopkins University Press, 2011). Ces interrogations ne sont d'ailleurs pas sans enjeux pour l'histoire du fait religieux et invitent à une redéfinition de ses contours et de sa méthodologie.

4 Guillaume Cuchet tire parti de l'abondante historiographie nord-américaine, tant sur les États-Unis que sur la France, tout en s'insérant dans la ligne des récents travaux réalisés, en particulier en France et en Italie, sur l'attitude de l'Église catholique à l'égard des nouvelles méthodes d'investigation des phénomènes psychiques. L'ouverture des archives du Saint-Office a en effet suscité une vague d'études sur le magnétisme, le spiritisme et l'hypnotisme. La découverte de ces archives, à l'occasion d'une thèse de doctorat, publiée en 2005 chez Armand Colin sous le titre Le Crépuscule du purgatoire (voir en particulier le chapitre 3 « Le défi spirite »), joue d'ailleurs un rôle 
décisif dans le choix de Cuchet de consacrer sa thèse d'habilitation à diriger des recherches à l'histoire du spiritisme (Faire de l'histoire dans une société sortie de la religion, Paris, Publications de la Sorbonne, 2013, p. 81).

5 L'auteur offre une lecture innovante du spiritisme français en proposant d'en faire l'observatoire des bouleversements sociaux qui traversent la société française du XIX siècle. Analysant le spiritisme français sous l'angle d'un "phénomène de société " (p. 18), il consacre son étude à ce qu'il appelle sa «grande époque » (p. 17 et 404), celle de la vogue des tables tournantes. Son étude s'étend donc des premières manifestations spirites aux États-Unis, en 1848, et de leur réception en France jusqu'à la montée des oppositions au mouvement, qui atteignent leur apogée avec le procès pour fraude mené contre le photographe spirite Édouard-Isidore Buguet en 1875. Les phases ultérieures du spiritisme - le développement des phénomènes médiumniques, et surtout l'affirmation des recherches psychiques, puis de la métapsychique - se trouvent hors de son champ d'investigation. L'ouvrage réalise une synthèse de travaux déjà publiés, tout en se référant à des sources imprimées originales. La bibliographie anglo-saxonne est dans l'ensemble bien maîtrisée. Une confrontation avec les thèses de M. Brady Brower (Unruly Spirits: The Science of Psychic Phenomena in Modern France, Urbana - Chicago Springfield, University of Illinois Press, 2010) qui, de manière pionnière, a examiné le spiritisme français sous le prisme des changements sociopolitiques, aurait été toutefois utile. Le recours aux archives est par contre limité.

6 L'histoire de la "grande époque » du spiritisme, que nous présente Cuchet, se déploie selon trois grands moments. Il y a tout d'abord la première phase d'acclimatation de l'«invention" américaine qui produit un effet de "mode» (parties 1 et 2). De phénomène de mode, le spiritisme devient progressivement un phénomène de société à la faveur de la constitution d'une doctrine, de la mise en place d'une organisation institutionnelle et, plus encore, de la propagation d'un «spiritisme culturel » qui dépasse les bornes strictes du mouvement spirite (parties 3, 4 et 5). L'auteur examine enfin les motifs du déclin que connaît ensuite le spiritisme en se penchant sur l'antispiritisme (partie 6).

7 Dans une première partie, Guillaume Cuchet examine le transfert de la pratique des tables tournantes qui s'opère entre France et États-Unis. Il retrace ainsi la naissance du spiritisme aux États-Unis, en mettant en lumière le contexte social dans lequel celui-ci émerge et en insistant sur son inscription dans un « renouveau religieux » plus large. L'auteur prête ensuite attention aux premières réceptions de ces pratiques, à travers les comptes rendus que la presse française, en particulier catholique, fait, à partir de 1852, des événements américains, avant d'examiner les débuts de la diffusion concrète de ces pratiques.

8 L'auteur s'intéresse ensuite à la manière dont ces pratiques exotiques suscitent un effet de «mode ». Il retrace ainsi les trois grandes étapes de l'évolution de la pratique des tables qui de simplement «tournantes» deviennent «parlantes» avant que les médiums n'imposent leur présence. Il examine également le rôle joué par la presse dans la diffusion de ces pratiques, avant de souligner comment la conjoncture politique et scientifique a contribué à amplifier le phénomène.

9 Comment passe-t-on d'un phénomène de "mode » à un phénomène de "société »? Cuchet situe ce passage en 1860, année de la deuxième édition du Livre des esprits d'Allan Kardec. La constitution de la croyance en « doctrine ", à travers la publication d'œuvres maîtresses, représente ainsi un facteur majeur de cette évolution. Soucieux 
de faire de l'œuvre de Kardec une entreprise collective, l'auteur se penche d'abord sur les travaux précurseurs, pour ensuite présenter le personnage de Kardec dans toute son épaisseur sociale, culturelle, politique et religieuse. Il examine enfin le contenu de la " révélation spirite ", accordant une place centrale à la doctrine de la réincarnation.

Cuchet met encore en valeur, dans sa quatrième partie, l'impulsion déterminante donnée par la constitution du spiritisme en mouvement organisé autour de 1860. Il y met tout d'abord en lumière le rôle joué par la libéralisation du régime impérial et la montée de l'intransigeantisme catholique qui provoque, d'une part, le ralliement des catholiques libéraux au mouvement spirite et, d'autre part, la montée d'une réaction antispirite qui assure une publicité involontaire au mouvement. En analysant les différentes composantes du mouvement spirite, l'auteur centre son attention sur Kardec du fait de son succès populaire. Le personnage de Camille Flammarion, dont le rayonnement a été également important, aurait sans doute mérité d'être plus amplement traité. Le chapitre 12 consacré à « La composition du mouvement » contient de très intéressants développements sur la diffusion du spiritisme en milieu ouvrier.

11 La cinquième partie du livre rend compte de l'émergence d'un "spiritisme culturel » qui se répand dans l'ensemble de la société française. Il fait ainsi du spiritisme la seule "philosophie religieuse » ayant réussi à se transformer en "mouvement populaire » (p. 282), ralliant en particulier des ex-républicains, déçus par la tournure autoritaire prise par le régime napoléonien. Le rapprochement, effectué dans le chapitre 16, entre spiritisme et « deuil romantique » est tout à fait suggestif.

12 Enfin, dans une dernière partie, l'auteur évoque la montée de l'opposition au spiritisme au cours des années 1860. Il souligne le rôle crucial joué par l'Église catholique dans cette lutte. Son attitude s'est progressivement durcie à mesure que l'intransigeantisme gagne du terrain, sans toutefois réussir à obtenir une condamnation formelle du spiritisme. Cuchet s'attache encore à mettre en lumière la diffusion d'un " antispiritisme laïque " alors que l'intense couverture médiatique des manifestations spirites les rend plus vulnérables aux accusations de fraude. Dans son chapitre conclusif, l'auteur s'interroge sur les causes du déclin du spiritisme comme "phénomène de société », qu'il situe autour de 1866. Il montre combien les scandales ont nui à l'image du spiritisme. Il suggère également que les atteintes à la liberté religieuse dues au rapprochement entre Église et Empire, de même que la montée du républicanisme athée, à la faveur du retour des proscrits, ont été pour une grande part dans cette désaffection.

13 En faisant du spiritisme un "phénomène de société ", l'auteur vise à redonner une légitimité culturelle et institutionnelle à l'histoire religieuse en mettant en valeur sa contribution à l'histoire sociale. Son propos s'inscrit dans une tendance lourde de l'histoire religieuse contemporaine en France. Cuchet se revendique d'une « histoire des croyances ", dans la ligne de Michel de Certeau et de Philippe Boutry notamment, où la croyance est considérée comme un observatoire privilégié des mutations sociales et culturelles. Le livre invite ainsi à un débat plus large sur les méthodes et problématiques de l'histoire du fait religieux contemporain.

14 Comme de récentes études l'ont bien mis au jour, l'irruption du spiritisme, aux côtés du magnétisme et de l'hypnose, a profondément bouleversé les rapports entre science et religion, faisant surgir un "troisième élément" aux contours flous et aux frontières fluctuantes (David Armando, "Spiriti e fluidi: Medicina e religione nei documenti del Sant'Uffizio sul magnetismo animale (1840-1856)", in Médecine et religion: compétitions, 
collaborations, conflits (XII-XXe siècles), Maria-Pia Donato et alii (dir.), Rome, Collection de l'École française de Rome, 2013, p. 195). Elle vient provoquer l'historien du religieux en l'incitant à prêter davantage attention au "caractère contesté " (Ann Taves, Fits, Trances, and Visions, p. 7) de l'expérience qu'il étudie. Expérience surnaturelle, fraude ou manifestation psychique: les interprétations divergent selon les interlocuteurs. Comment prendre en compte et, également, rendre compte tant de l'instabilité de l'objet étudié que de la subjectivité des acteurs et de l'historien lui-même aux prises avec l'étude de ces phénomènes? Une réflexion épistémologique approfondie serait dès lors nécessaire afin d'aborder avec rigueur scientifique et modestie ce nouveau champ de recherches. Un tel travail, certes austère mais très bénéfique, offrirait des clés importantes pour repenser l'histoire religieuse dans le contexte contemporain de pluralisation du « religieux ». Il profiterait de l'apport des discussions actuelles autour du statut et des méthodes des religious studies (cf. Ann Taves, Religious Experience Reconsidered: A Building-Block Approach to the Study of Religion and Other Special Things, Princeton, Princeton University Press, 2011).

L'histoire du spiritisme représente en effet un poste d'analyse particulièrement fructueux de la mutation du régime de la " croyance » au XIX $x^{e}$ siècle. La pluralisation du religieux s'opère sur fond d'affirmation de la subjectivité. L'équilibre fragile entre science et religion, gagné de dure lutte au temps des Lumières, se trouve remis en question. Dans cette perspective, un rapprochement entre histoire des sciences, histoire religieuse et histoire sociale et culturelle est tout à fait crucial. Dès lors, on regrettera que l'auteur n'ait pas davantage tiré parti des discussions particulièrement riches menées par les historiens des sciences autour du spiritisme. L'affirmation selon laquelle "catholiques et laïques se sont objectivement entendus pour limiter le pluralisme religieux » (p.403) mériterait une argumentation plus serrée. De même, la question de la perméabilité des religions monothéistes aux nouvelles formes de religiosité, évoquée rapidement en termes de «spiritisme latent» (p. 295 et p. 297), nécessiterait des développements plus précis.

16 Faire l'histoire religieuse de ce nouveau régime de "croyance » représente un défi de taille pour l'historien du religieux, traditionnellement plus coutumier des formes institutionnalisées de l'expérience religieuse. Défi d'abord au niveau des sources utilisées, mais aussi, et par voie de conséquence, au niveau du point de vue adopté par l'historien. La position de quasi-monopole que l'Église catholique a sur la sphère du religieux dans la France du xIX ${ }^{e}$ siècle n'est en effet pas indifférente. Les sources catholiques, archivistiques comme imprimées, tiennent ainsi une place prééminente dans le travail de Cuchet. L'histoire du spiritisme qu'il nous propose apparait dès lors davantage comme une histoire des observateurs catholiques et, dans une moindre mesure, laïcs du phénomène, que comme une histoire du mouvement spirite lui-même dans ses dynamiques internes. Dans cette optique, on s'interrogera sur la pertinence de l'assimilation du mouvement spirite à un courant «hétérodoxe » (p. 20, 40, 43 et 137 notamment). Ce qualificatif n'induit-il pas un jugement de valeur sur ces nouvelles croyances? L'auteur veut-il, au contraire, suggérer que le spiritisme français est un surgeon du catholicisme? Une confrontation avec l'historiographie américaine et, en particulier, avec les travaux de John Warne Monroe, où ce qualificatif a été forgé, serait à cet égard tout à fait précieuse. Elle permettrait de comparer très utilement la manière dont protestantisme et catholicisme ont composé avec l'apparition de ce nouveau régime de «croyance » et de mettre en lumière également comment les conceptions 
distinctes de rapport entre Église et État, développées en France et aux États-Unis, influent sur la gestion de ces nouvelles «croyances». Il serait en outre possible d'élargir l'enquête côté catholique en proposant une analyse exhaustive du fameux dossier du Saint-Office sur le magnétisme, le spiritisme et l'hypnose. Si le dossier a suscité l'attention de multiples historiens, qui lui ont consacré des études partiales, généralement limitées à un seul des phénomènes, il n'a jamais été examiné dans son ensemble.

17 Enfin, il conviendrait de réfléchir à nouveaux frais sur l'impact du contexte religieux actuel sur la recherche en histoire religieuse contemporaine. Le décrochage du catholicisme dans la société française autour des années 1960-1980 constitue le trait saillant de l'histoire que Cuchet s'efforce de construire. Avec justesse, il fait le pari d'une histoire longue de cette « rupture » dont il éclaire ici un moment phare. Il serait toutefois pertinent de prolonger l'entreprise afin de mettre davantage en lumière les dynamiques contradictoires de ce décrochage, entre crispation et ouverture, recul conservateur et avancées sociales, sécularisation et réinvestissement de l'identité religieuse, rejet et accueil du nouveau régime de "croyance ». Une telle approche aurait le mérite de proposer une périodisation plus précise de cette évolution et d'éviter des rapprochements entre phénomènes passés et situation actuelle, sources de possibles ambiguïtés. Une grande prudence méthodologique et épistémologique s'impose lorsqu'il s'agit de généraliser les résultats de l'enquête historique en vue de réaliser une « histoire globale à partir d'une croyance » (p. 18). 